\title{
MicroRNA-545 targets ZEB2 to inhibit the development of non-small cell lung cancer by inactivating Wnt/及-catenin pathway
}

\author{
JIANYING CUI $^{1}$, GUODONG PAN ${ }^{2}$, QINGJUAN HE ${ }^{3}$, LIZHI YIN ${ }^{4}$, RUI GUO $^{5}$ and HONGMEI BI ${ }^{6}$ \\ ${ }^{1}$ Department of Respiratory Medicine, Anqiu People's Hospital, Weifang, Shandong 262100; \\ ${ }^{2}$ Department of Thoracic Surgery, Traditional Chinese Medical Hospital of Huangdao District, Qingdao, \\ Shandong 266500; ${ }^{3}$ Department of Respiratory Medicine, The People's Hospital of Zhangqiu Area; \\ ${ }^{4}$ Health Management Center, The People's Hospital of Zhangqiu Area; ${ }^{5}$ Outpatient Department, \\ The People's Hospital of Zhangqiu Area, Jinan, Shandong 250200; ${ }^{6}$ Department of Respiratory Medicine, \\ The Third People's Hospital of Qingdao, Qingdao, Shandong 266041, P.R. China
}

Received March 1, 2019; Accepted June 10, 2019

DOI: $10.3892 / 01.2019 .10619$

\begin{abstract}
The specific function of microRNA-545 (miR-545) has been reported to regulate the development of human cancers. However, the effect of miR-545 is still unclear in non-small cell lung cancer (NSCLC). Hence, this study explored the molecular mechanism of miR-545 in NSCLC The expression levels of miR-545 and ZEB2 were measured through reverse transcription-quantitative polymerase chain reaction (RT-qPCR) assay. The protein expression was detected by western blotting. Dual luciferase assay was applied to evaluate the relationship between miR-545 and zinc finger E-box-binding homeobox 2 (ZEB2). MTT and Transwell assays were used to investigate the function of miR-545 in NSCLC. The expression of miR-545 was decreased in NSCLC tissues. The overexpression of miR-545 suppressed the migration, invasion and proliferation of NSCLC cells. Furthermore, ZEB2 was a direct target gene of miR-545. The knockout of ZEB2 suppressed the development of NSCLC. miR-545 inhibited the progression of NSCLC through targeting ZEB2. Moreover, miR-545 repressed the development of NSCLC via blocking EMT and Wnt/ $\beta$-catenin pathway. In conclusion, miR-545 inhibited the progression of NSCLC through targeting ZEB2 and blocking EMT and Wnt/ $\beta$-catenin pathway.
\end{abstract}

Correspondence to: Dr Hongmei Bi, Department of Respiratory Medicine, The Third People's Hospital of Qingdao, 29 Yongping Road, Licang, Qingdao, Shandong 266041, P.R. China

E-mail: fd617339ug@163.com

Key words: miR-545, non-small cell lung cancer, ZEB2, Wnt $/ \beta$-catenin pathway

\section{Introduction}

With the increase of aging population, the acceleration of industrialization, and the intensification of environmental pollution, the incidence and mortality of lung cancer has increased rapidly (1). Lung cancer has become one of the most rapidly increasing cancers in China, and will be the leading cause of death for a long time (2). Therefore, the prevention and early detection of lung cancer is expected to become one of the key points of tumor control in China. Moreover, non-small cell lung cancer (NSCLC) accounts for approximately $84 \%$ of all lung cancers, which can be transferred to the whole body through lymphoids and blood. NSCLC is metastatic but not infectious (3). It should be emphasized that the clinical staging before the treatment of NSCLC is crucial. Accurate clinical staging is not only helpful for doctors to make scientific and reasonable treatment program for patients with NSCLC, but also can better help doctors to judge the prognosis of NSCLC and modify the auxiliary treatment strategies after surgery (4). However, there is a great disparity in clinical diagnosis and treatment due to the non-standard diagnosis and treatment of NSCLC.

Carcinogenesis of NSCLC involves in multistep process which is regulated by activating oncogenes or inactivating tumor-suppressing genes (5). Recently, several microRNAs (miRNAs) have been reported to participate in tumorigenesis of NSCLC which play a carcinogenic or suppressive role in the progression of NSCLC. For example, miR-520a inhibited cell growth and metastasis of NSCLC through PI3K/AKT/mTOR signaling pathway (6). On the contrary, miR-96 promoted invasion and metastasis by targeting GPC3 in NSCLC cells (7). The function of microRNA-545 (miR-545)in human disease and cancers has drawn increased attention. The downregulation of miR-545 has been identified in pancreatic ductal adenocarcinoma which inhibited tumor growth through targeting RIG1 (8). In addition, miR-545 can be used as an early biomarker of Alzheimer's disease (9). Moreover, 
a previous study reported the role of miR-545 in lung cancer. miR-545 arrested cell cycle and induced cell apoptosis in lung cancer through regulating cyclin D1 and CDK4 (10). However, the effect of miR-545 on the development of NSCLC is still unknown.

Zinc finger E-box-binding homeobox 2 (ZEB2) has been found to participate in pathogenesis of human cancers (11). ZEB2 has been documented to be correlated with metastasis and invasion in several types of malignant tumors. Chua et al (12) demonstrated that ZEB2 repressed E-cadherin expression and enhanced epithelial to mesenchymal transition (EMT) of mammary epithelial cells. Furthermore, miR-132 suppressed the migration and invasion of lung cancer cells via targeting the EMT regulator ZEB2 (13). However, the relationship of miR-545 with ZEB2 still needs to be investigated in NSCLC. ZEB2 has been revealed to be involved in the Wnt/ $\beta$-catenin pathway to regulate the development of gastric adenocarcinoma (14). In lung cancer, miR-130b targeted PTEN to induce resistance to cisplatin by activating $\mathrm{Wnt} / \beta$-catenin pathway (15). However, no interaction between ZEB2 and Wnt/ $\beta$-catenin pathway in NSCLC has been proposed.

The main objective of this research was to observe the aberrant expression, as well as the function of miR-545 in the progression of NSCLC with the aim to provide a theoretical basis for illuminating the regulatory mechanism of miR-545 in NSCLC.

\section{Patients and methods}

Clinical tissues. In total, 84 human NSCLC tissues and adjacent normal lung tissues were acquired from the The Third People's Hospital of Qingdao (Qingdao, China). The patients with NSCLC did not receive any treatment before the operation. The tissues were frozen in liquid nitrogen and then stored at $-80^{\circ} \mathrm{C}$ in a refrigerator. All the patients provided informed consent and this experiment was approved by the Institutional Ethics Committee of The Third People's Hospital of Qingdao.

Cell culture.H522 [cat. no.ZK156(XR)], A549 [cat. no ZK0930 (XR)], H650 [cat. no.ZK1573(XR)] cell lines and human normal bronchial epithelial cells (HBECs) (ZKCC-X2676) were used for this experiment. The cell lines were acquired from Shanghai Maisha Biotechnology. The cells were seeded in DMEM medium with $10 \%$ fetal bovine serum (FBS) and cultured at $37^{\circ} \mathrm{C}$ with $5 \% \mathrm{CO}_{2}$.

Cell transfection. The miR-545 mimics or inhibitor and negative control (NC) were obtained from RiboBio. ZEB2 siRNA or negative control siRNA was purchased from Genechem. They were transferred into A549 cells respectively by using Lipofectamine 2000 (Invitrogen; Thermo Fisher Scientific, Inc.) based on the manufacturer's protocol.

Reverse transcription-quantitative polymerase chain reaction (RT-qPCR). TRIzol reagent (Invitrogen; Thermo Fisher Scientific, Inc.) was applied to extract total RNA in NSCLC. The synthesis of cDNA was performed using RevertAid First Strand cDNA kit (cat. no. K1622; Thermo Fisher Scientific, Inc.). RT-qPCR was conducted using SYBR-Green Reagent (cat. no. 4385612; Thermo Fisher Scientific, Inc.) on 7500
Fast Real-Time PCR system (Thermo Fisher Scientific, Inc.). The thermocycling parameters were as follows: $95^{\circ} \mathrm{C}$ for $3 \mathrm{~min}$ and 38 cycles of $95^{\circ} \mathrm{C}$ for $15 \mathrm{sec}$ followed by $60^{\circ} \mathrm{C}$ for $30 \mathrm{sec}$ The temperature conditions of the reverse transcription were as follows: $37^{\circ} \mathrm{C}$ for $15 \mathrm{~min}$ and $85^{\circ} \mathrm{C}$ for $5 \mathrm{sec}$. U6 or GAPDH was used as internal control for miR-545 or ZEB2. Their expression levels were calculated using the $2^{-\Delta \Delta \mathrm{Cq}}$ method (16).

Western blot analysis. The protein samples were obtained using RIPA lysis buffer. Protein determination method was calculated using bicinchoninic acid (BCA). Then equal amounts of proteins $(30 \mu \mathrm{g})$ were separated through a $10 \%$ SDS-PAGE and transferred into a polyvinylidene difluoride (PVDF) membrane (Thermo Fisher Scientific, Inc.). Then, the membranes were blocked with $5 \%$ non-fat milk for $1 \mathrm{~h}$ at room temperature. Next the membranes were incubated overnight at $4^{\circ} \mathrm{C}$ with EMT markers [(E-cadherin (rabbit monoclonal antibody; dilution, 1:1,000; cat. no. ab1416; Abcam), N-cadherin (rabbit polyclonal antibody; dilution, 1:1,000; cat. no. 18203; Abcam), vimentin (rabbit polyclonal antibody; dilution, 1:1,000; cat. no. 137321; Abcam), Wnt/ $\beta$-catenin pathway markers ( $\beta$-catenin), rabbit polyclonal antibody; dilution, 1:1,000; cat. no. 6302; Abcam), ZEB2 (rabbit polyclonal antibody; dilution, 1:1,000; cat. no. 138222; Abcam)] and GAPDH primary, rabbit monoclonal antibodies (dilution, 1:1,000; cat. no. ab181602; Abcam). After washing, they were incubated with the corresponding secondary mouse anti-rabbit (dilution, 1:2,000; cat. no. 3678; Abcam) or goat anti-mouse (dilution, 1:2,000; cat. no. 58802; Abcam) antibodies conjugated with horseradish peroxidase (Cell Signaling Technology, Inc.) for $2 \mathrm{~h}$ at room temperature. Then, protein expression levels were measured by ECL (Pierce; Thermo Fisher Scientific, Inc.).

MTT assay. MTT (3-(4,5-dimethylthiazol-2-yl)-2,5-diphenyltetrazolium bromide) assay was used to assess cell viability. The $5 \times 10^{4}$ A549 cells with transfection were incubated in a 96-well plate at $37^{\circ} \mathrm{C}$ for $48 \mathrm{~h}$. Next, the cells were incubated at $37^{\circ} \mathrm{C}$ in a serum-free medium with $20 \mu \mathrm{l}$ MTT for $4 \mathrm{~h}$. Finally, they were dissolved in $100 \mu \mathrm{l}$ of dimethyl sulfoxide after removing the supernatant and absorbance was read at $490 \mathrm{~nm}$ on a microplate spectrophotometer (Thermo Labsystems).

Transwell assay. Transwell chambers ( $8 \mu \mathrm{m}$ pore size; Millipore) were applied to evaluate the migratory and invasive ability of A549 cells in 24-well plates. A549 cells $\left(3 \times 10^{4}\right)$ without serum were put in the upper chamber on the non-coated membrane, and the lower chamber was filled with $10 \%$ FBS to induce A549 cells to migrate or invade through the membrane. The cells were put in the upper chamber with $100 \mu \mathrm{l}$ Matrigel (dilution, 1:8 in DMEM medium) for invasion assay. The cells on the lower surface of the membrane were fixed with $4 \%$ paraformaldehyde, stained with $1 \%$ crystal violet (Solarbio) for $5 \mathrm{~min}$ and subsequently counted in 3 randomly selected fields under an inverted microscope (Leica Microsystems $\mathrm{GmbH}$ ) at magnification x200.

Dual luciferase assay. The wild or mutant type of 3'-UTR of ZEB2 was inserted into pcDNA3.1 plasmid vector (Promega Corporation) to perform luciferase reporter experiments. Then, 
Table I. Relationship of miR-545 expression and their clinicopathological characteristics of NSCLC patients.

\begin{tabular}{|c|c|c|c|c|}
\hline \multirow[b]{2}{*}{ Characteristics } & \multirow[b]{2}{*}{ Cases } & \multicolumn{2}{|c|}{ miR-545 } & \multirow[b]{2}{*}{ P-value } \\
\hline & & High & Low & \\
\hline Age (years) & & & & 0.072 \\
\hline$\geq 60$ & 54 & 20 & 34 & \\
\hline$<60$ & 30 & 12 & 18 & \\
\hline Sex & & & & 0.21 \\
\hline Male & 50 & 21 & 29 & \\
\hline Female & 34 & 16 & 18 & \\
\hline Tumor size (mm) & & & & 0.144 \\
\hline$\leq 3$ & 48 & 22 & 26 & \\
\hline$>3$ & 36 & 15 & 21 & \\
\hline Lymph node metastasis & & & & $0.016^{\mathrm{a}}$ \\
\hline Yes & 19 & 7 & 12 & \\
\hline No & 65 & 25 & 40 & \\
\hline Tumor stage & & & & $0.02^{\mathrm{a}}$ \\
\hline I-II & 60 & 24 & 36 & \\
\hline III-IV & 24 & 10 & 14 & \\
\hline
\end{tabular}

Statistical analyses were performed by the $\chi^{2}$ test. ${ }^{\text {a }}<0.05$ was considered statistically significant. miR-545, microRNA-545; NSCLC, non-small cell lung cancer.

wild or mutant type of 3'-UTR of ZEB2 and miR-545 mimic were transfected into A549 cells. Subsequently, the luciferase activity was measured through dual luciferase assay system (Promega Corporation).

Statistical analysis. The data are shown as mean \pm SD. SPSS 19.0 or Graphpad Prism 6 (GraphPad Software, Inc.) was employed to analyze these data. The difference between the groups was calculated through one-way ANOVA followed by the Bonferroni post hoc test. The correlation of miR-545 with clinicopathological characteristics of NSCLC was calculated through the Chi-squared test. The overall survival rates and survival differences were detected through univariate Kaplan-Meier method followed by log-rank test. $\mathrm{P}<0.05$ was considered to indicate a statistically significant difference.

\section{Results}

Expression of miR-545 is decreased in NSCLC tissues. Alteration of miR-545 expression was examined in NSCLC tissues by RT-qPCR assay. miR-545 was found to be downregulated in NSCLC tissues in comparison with the normal tissues $(\mathrm{P}<0.01$, Fig. 1A). Moreover, the close association between the decreased expression of miR-545 and lymph node metastasis $(\mathrm{P}=0.016)$ or tumor stage $(\mathrm{P}=0.02)$ was identified in NSCLC patients (Table I). Low miR-545 expression predicted worse prognosis and was associated with shorter overall survival of NSCLC patients $(\mathrm{P}=0.044$, Fig. 1B). Thus, it was implied that miR-545 was involved in the tumorigenesis and prognosis of NSCLC.
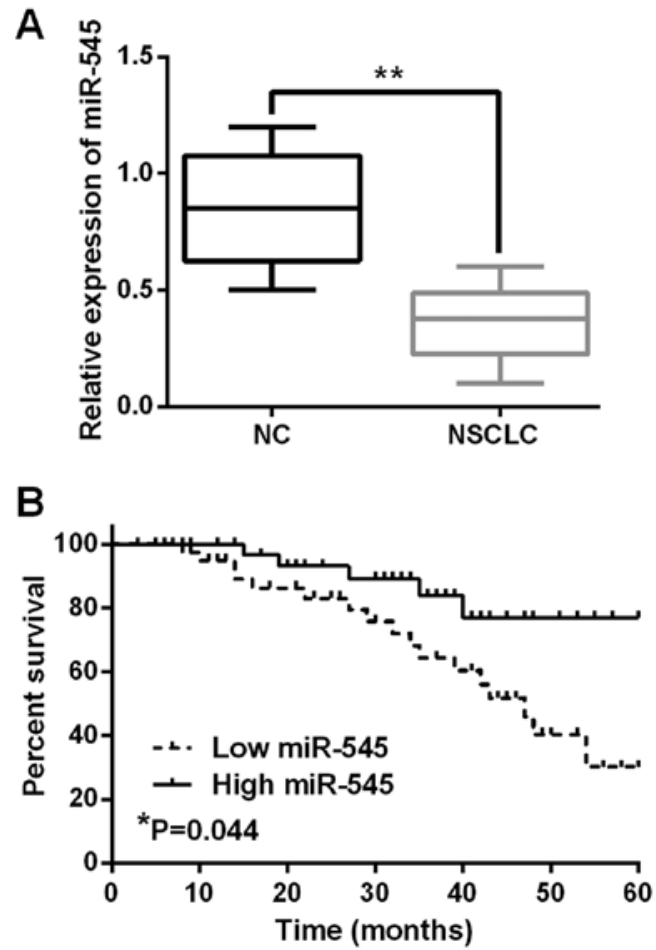

Figure 1. Expression of miR-545 is decreased in NSCLC tissues. (A) The expression levels of miR-545 in NSCLC tissues. (B) The low miR-545 expression was associated with shorter overall survival of NSCLC patients. ${ }^{*} \mathrm{P}<0.05,{ }^{* *} \mathrm{P}<0.01$. miR-545, microRNA-545; NSCLC, non-small cell lung cancer. NC, negative control.

Overexpression of miR-545 inhibits progression of NSCLC. The expression levels of miR-545 were observed in H522, A549, H650 and HBECs cell lines. Decreased miR-545 expression was identified in H522, A549 and H650 cell lines compared with HBEC cells ( $\mathrm{P}<0.05$ or 0.01 , Fig. $2 \mathrm{~A})$. In order to investigate the function of miR-545 in NSCLC, A549 cells were transfected with miR-545 mimics or inhibitor. The RT-qPCR assay showed that miR-545 expression level was enhanced by miR-545 mimics and inhibited by miR-545 inhibitor ( $\mathrm{P}<0.01$, Fig. 2B). MTT assay suggested that upregulation of miR-545 inhibits the proliferation of A549 cells $(\mathrm{P}<0.01$, Fig. 2C), whereas, cell proliferation was promoted by the knockout of miR-545 in NSCLC ( $\mathrm{P}<0.05$, Fig. 2D). Similarly, cell migration was also suppressed by upregulation of miR-545 and promoted by the knockout of miR-545 in A549 cells ( $\mathrm{P}<0.01$, Fig. 2E). Cell invasion in A549 cells was also identified with similar results $(\mathrm{P}<0.01$, Fig. $2 \mathrm{~F})$. These results indicate that miR-545 plays a suppressive role in the progression of NSCLC.

ZEB2 is a direct target gene of miR-545. Furthermore, miR-545 was predicted to have binding sites with the 3'-UTR of ZEB2 in the database of TargetScan (http://www.targetscan. org/) (Fig. 3A). Then luciferase reporter assay was performed to confirm the above prediction. As expected, the luciferase activity of Wt-ZEB2 was obviously inhibited by miR-545 mimics $(\mathrm{P}<0.01$, Fig. 3B). However, the luciferase activity of Mut-ZEB2 was not affected by miR-545 mimics. Moreover, the negative association between miR-545 and ZEB2 was detected in NSCLC tissues $\left(\mathrm{P}<0.0001, \mathrm{R}^{2}=0.826\right.$; Fig. $\left.3 \mathrm{C}\right)$. 
A

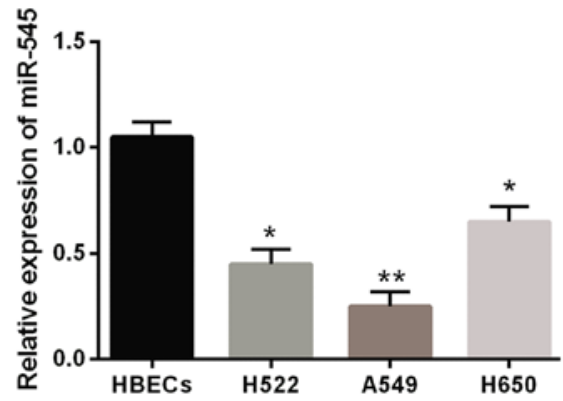

C

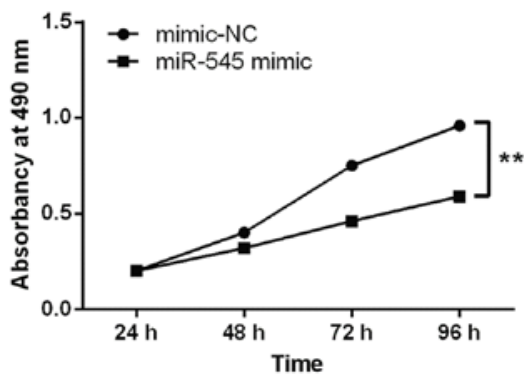

E

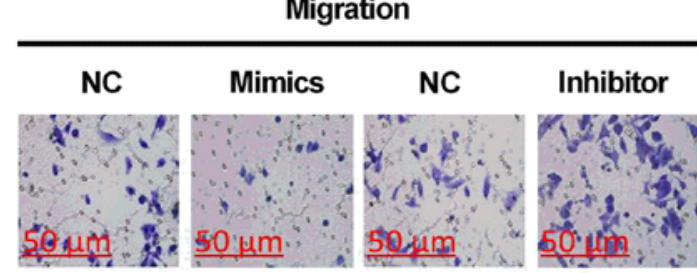

$\mathbf{F}$

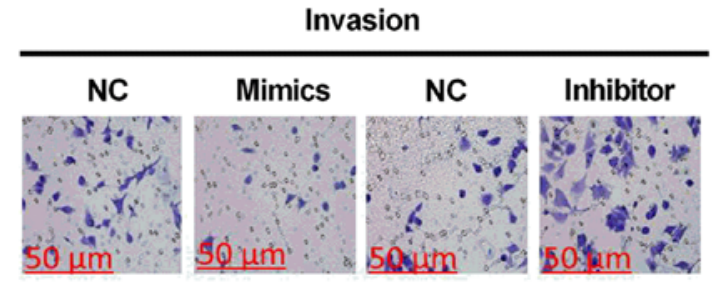

B

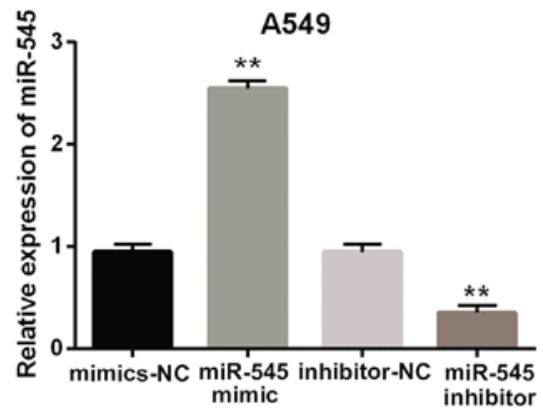

D
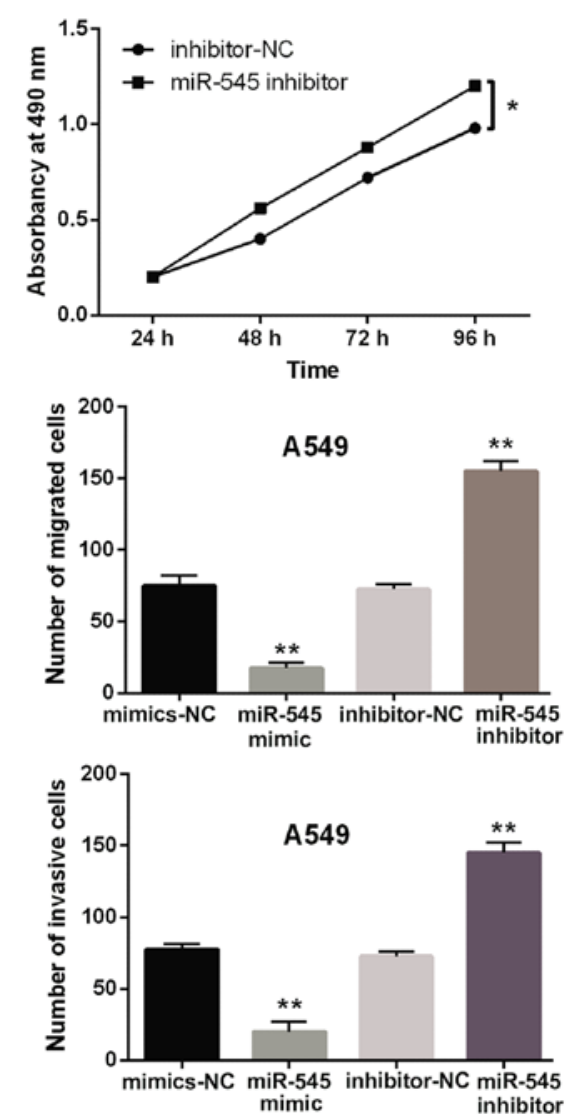

Figure 2. Overexpression of miR-545 inhibits the progression of NSCLC. (A) The expression of miR-545 in H522, A549, H650 and HBECs cell lines. (B) The expression of miR-545 was examined in A549 cells with miR-545 mimics or inhibitor. (C and D) Cell proliferation was measured in cells containing miR-545 mimics or inhibitor. (E and F) Cell migration and invasion analysis in cells containing miR-545 mimics or inhibitor. ${ }^{*} \mathrm{P}<0.05$, ${ }^{* * *} \mathrm{P}<0.01$. miR-545, microRNA-545; NSCLC, non-small cell lung cancer; NC, negative control.

A

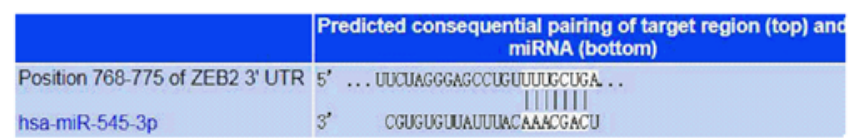

C

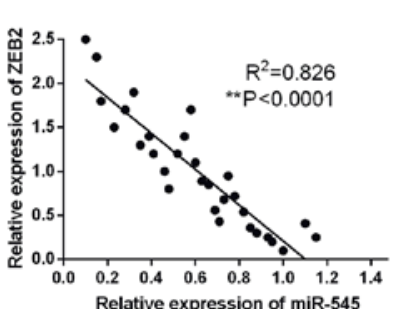

D

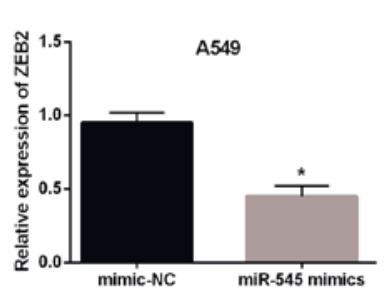

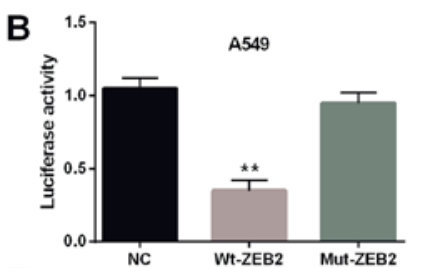

E

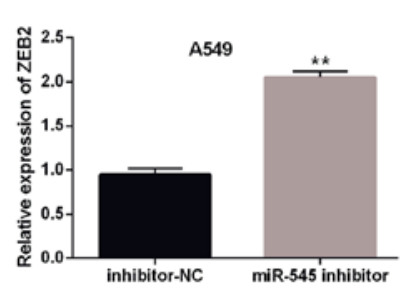

Figure 3. ZEB2 is a direct target gene of miR-545. (A) ZEB2 has binding sites with miR-545. (B) Luciferase reporter assay, (C) miR-545 has negative correlation with ZEB2. (D and E) The expression of ZEB2 were observed in A549 cells containing miR-545 mimics or inhibitor. "P<0.05, ${ }^{* *} \mathrm{P}<0.01$. ZEB2, zinc finger E-box-binding homeobox 2; miR-545, microRNA-545; NC, negative control. 
A

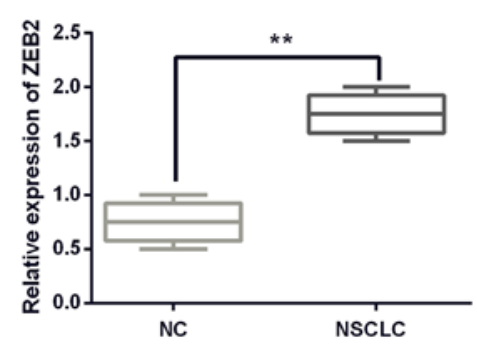

C

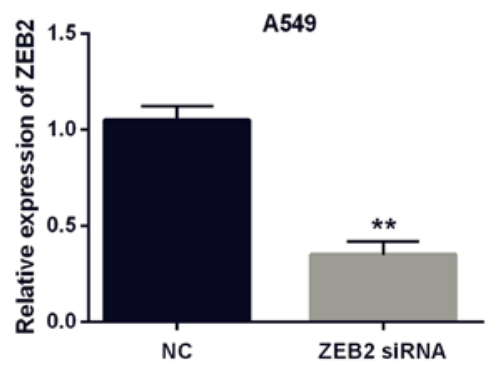

E

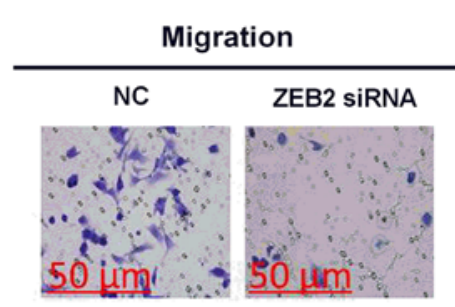

F

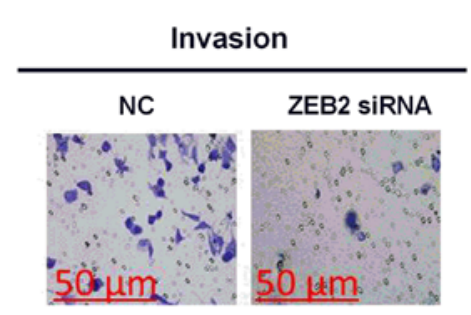

B

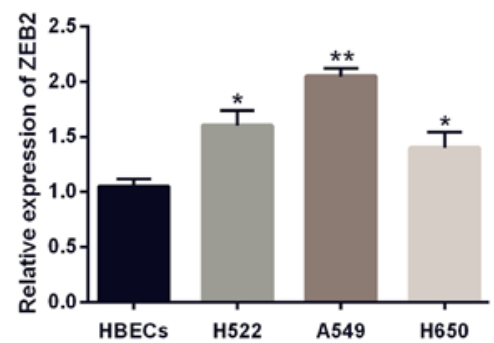

D
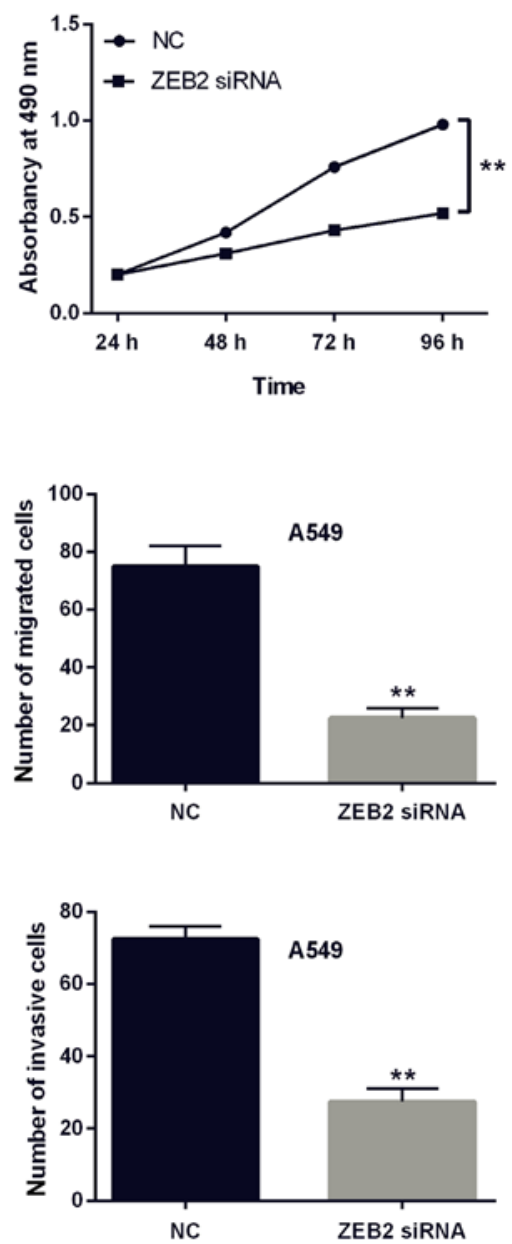

Figure 4. Knockout of ZEB2 suppresses the development of NSCLC. (A) The expression levels of ZEB2 in NSCLC tissues. (B) ZEB2 expression in H522, A549, H650 and HBECs cell lines. (C) The expression of ZEB2 was examined in A549 cells with ZEB2 siRNA. (D) Cell proliferation was measured in cells containing ZEB2 siRNA. (E and F) Migration and invasion analysis in cells containing ZEB2 siRNA. ${ }^{*} \mathrm{P}<0.05$, ${ }^{* *} \mathrm{P}<0.01$. ZEB2, zinc finger E-box-binding homeobox 2; NSCLC, non-small cell lung cancer; NC, negative control.

To further confirm their relationship, ZEB2 expression was examined in A549 cells with miR-545 mimics or inhibitor. Consistently, the expression level of ZEB2 was declined by miR-545 mimics $(\mathrm{P}<0.05$, Fig. 3D) and was enhanced by miR-545 inhibitor $(\mathrm{P}<0.01$, Fig. 3E). These results confirmed that miR-495 directly targeted ZEB2 and had negative association with ZEB2 expression in NSCLC tissues.

The knockout of ZEB2 suppresses the development of NSCLC. Subsequently, the expression of ZEB2 was identified in NSCLC tissues and cell lines. We found that ZEB2 was upregulated in NSCLC tissues in contrast to the normal tissues $(\mathrm{P}<0.01$, Fig. 4A). Increased ZEB2 expression was also identified in
H522, A549 and H650 cell lines compared with HBECs cells ( $\mathrm{P}<0.05$ or 0.01 , Fig. 4B). ZEB2 siRNA was transfected into A549 cells to investigate its role in NSCLC. The expression of ZEB2 was significantly declined by ZEB2 siRNA $(\mathrm{P}<0.01$, Fig. 4C). Furthermore, knockout of ZEB2 suppressed the proliferation of A549 cells ( $\mathrm{P}<0.01$, Fig. 4D). Moreover, cell migration and invasion were also found to be inhibited by ZEB2 siRNA ( $\mathrm{P}<0.01$, Fig. 4E and $\mathrm{F})$. These findings revealed that ZEB2 had carcinogenic effect on NSCLC.

miR-545 inhibits progression of NSCLC through targeting ZEB2. miR-545 mimics and ZEB2 vector were co-transfected into A549 cells to evaluate their interaction. It was found 
A

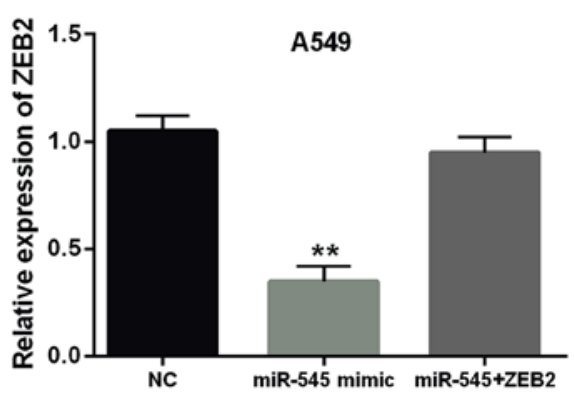

C

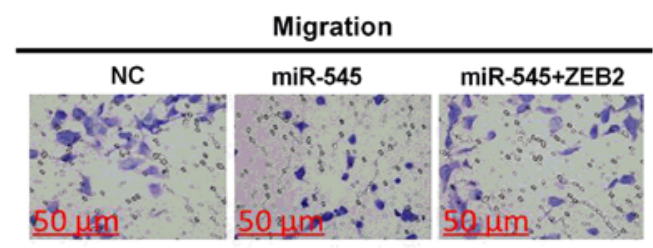

D

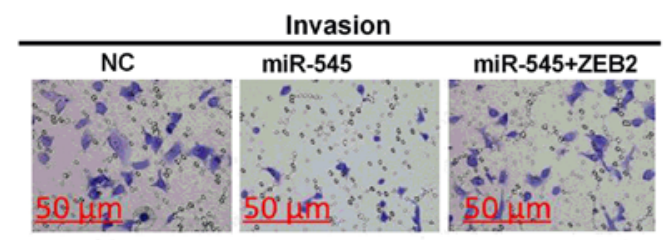

B
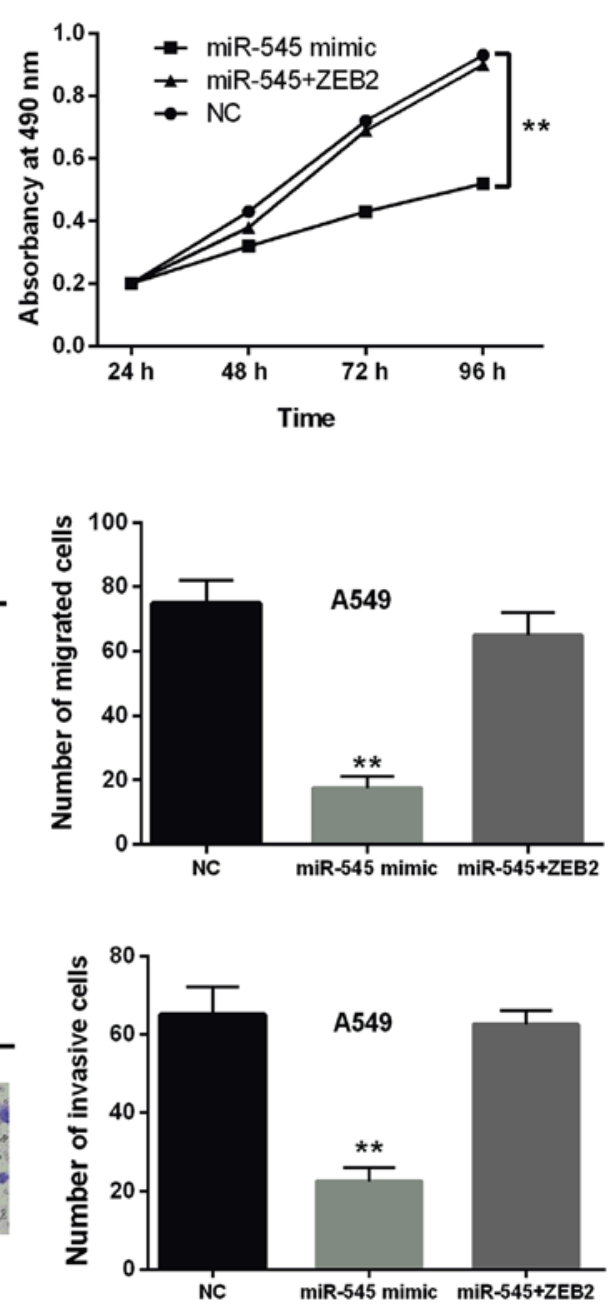

Figure 5. miR-545 inhibits progression of NSCLC through targeting ZEB2. (A) The expression of ZEB2 was measured in A549 cells with ZEB2 vector and miR-545 mimics. (B) Cell proliferation was measured in A549 cells with ZEB2 vector and miR-545 mimics. (C and D) Cell migration and invasion in A549 cells with ZEB2 vector and miR-545 mimics. ${ }^{* *} \mathrm{P}<0.01$. miR-545, microRNA-545; NSCLC, non-small cell lung cancer. NC, negative control; ZEB2, zinc finger E-box-binding homeobox 2.

that the decreased ZEB2 expression induced by miR-545 mimics was restored by ZEB2 vector in A549 cells $(\mathrm{P}<0.01$, Fig. 5A). Similarly, the inhibitory effect of miR-545 for cell proliferation was impaired by ZEB2 vector (P<0.01, Fig. 5B). Moreover, the diminished role of miR-545 for cell migration and invasion was also impeded by ZEB2 vector in NSCLC $(\mathrm{P}<0.01$, Fig. $5 \mathrm{C}$ and $\mathrm{D})$. Collectively, miR-545 inhibited the progression of NSCLC through targeting ZEB2.

miR-545 blocks EMT and Wnt/ $\beta$-catenin pathway in NSCLC. In order to further confirm the effect of miR-545 on cell survival and metastasis, EMT and $\mathrm{Wnt} / \beta$-catenin pathway was investigated in NSCLC. It was found that the expression levels of N-cadherin and vimentin were inhibited by overexpression of miR-545 which promoted E-cadherin expression in A549 cells (Fig. 6). Whereas, the knockout of miR-545 had opposite effect on the expression levels of the three markers (Fig. 6). The results suggested that miR-545 inhibited the metastasis of NSCLC cells through blocking EMT. Moreover, expression of $\beta$-catenin involved in Wnt/ $\beta$-catenin pathway was also inhibited by overexpression of miR-545 (Fig. 6), while the knockout

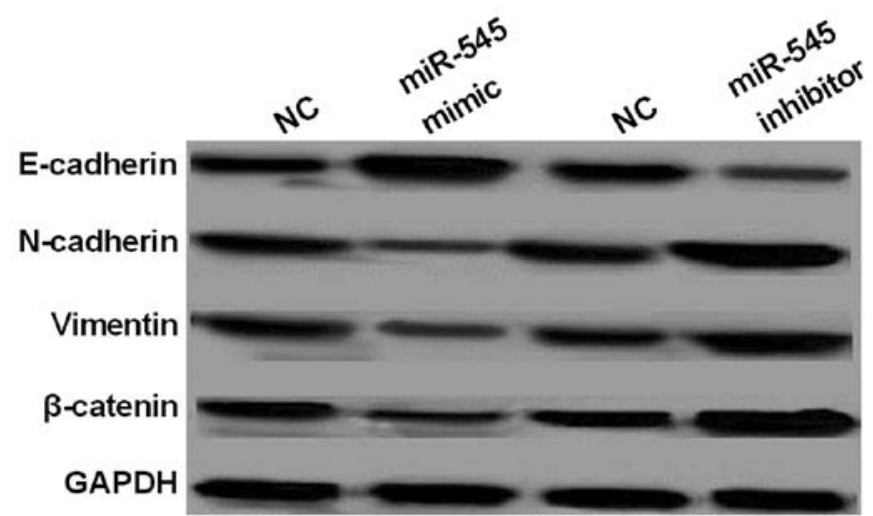

Figure 6. miR-545 blocks EMT and Wnt/ $\beta$-catenin pathway in NSCLC. Western blot analysis of E-cadherin, $\mathrm{N}$-cadherin, vimentin, ZEB2 and $\beta$-catenin in A549 cells contained miR-545 mimics or inhibitor. miR-545, microRNA-545; NSCLC, non-small cell lung cancer; NC, negative control.

of miR-545 promoted $\beta$-catenin expression (Fig. 6). In total, miR-545 repressed cell survival and metastasis through blocking EMT and Wnt/ $\beta$-catenin pathway in NSCLC. 


\section{Discussion}

Recently, exploiting and antagonizing microRNA regulation have been reported in therapeutic and experimental applications (17). Moreover, many miRNAs have been found to contribute or attenuate the carcinogenic effect of NSCLC, such as miR-101 (18) and miR-4443 (19). In the current study, we paid attention to the abnormal expression and function of miR-545. miR-545 was identified to be downregulated in NSCLC which was associated with worse prognosis of NSCLC patients. Similarly, Liao et al (20) found downregulation of miR-545 in Lewis lung carcinoma. In addition, miR-545 was related to poor prognosis of HCC patients shortening the 5-year overall survival (OS) (21). MTT and Transwell assays were performed to further explore the function of miR-545 in NSCLC. We found that overexpression of miR-545 inhibited the proliferation, migration and invasion of NSCLC cells. A previous study reported the tumor suppressing role of miR-545 in epithelial ovarian cancer (22). miR-545 negatively mediated colorectal cancer cell proliferation through upregulating EGFR expression (23). In the present study, miR-545 inhibited the progression of NSCLC through targeting ZEB2.

Previous studies have implied that ZEB2 was a vital oncogene which contributed to the progression of multiple cancers including NSCLC (24). Here, upregulation of ZEB2 was identified in NSCLC and the knockout of ZEB2 suppressed the development of NSCLC. Similar results of ZEB2 was also detected in osteosarcoma (25). miR-200c inhibited metastasis of NSCLC cells by targeting ZEB2 (26) which was consistent with our findings. Additionally, ZEB2 was reported to induce EMT via suppressing E-cadherin and promoting tumorigenesis (27). EMT is recognized as a crucial biological process which is related to metastasis of cancers. This study indicates that miR-545/ZEB2 axis blocked EMT to metastasis of NSCLC. Shi et al (28) demonstrated that downregulation of miR-218 contributed to EMT and tumor metastasis in lung cancer by targeting ZEB2. ZEB2 has been reported to mediate multiple pathways regulating cell proliferation, migration, invasion, and apoptosis in glioma, including Wnt/ $\beta$-catenin pathway (29). We also observed that miR-545/ZEB2 blocked Wnt/ $\beta$-catenin pathway to regulate the progression of NSCLC.

Accumulated evidence demonstrate that aberrant activation of the $\mathrm{Wnt} / \beta$-catenin pathway could result in tumor formation (30). Previous studies demonstrated that $\mathrm{Wnt} / \beta$-catenin pathway was involved in pathogenesis of NSCLC (31). Qi et al (29) revealed that downregulation of ZEB2 suppressed cell proliferation and reduced $\beta$-catenin expression. It is well known that $\beta$-catenin is a key regulator in $\mathrm{Wnt} / \beta$-catenin pathway. In the present study, overexpression of miR-545 declined $\beta$-catenin expression and miR-545 inhibited the development of NSCLC by targeting ZEB2 and inactivating Wnt/ $\beta$-catenin pathway. Similarly, miR-145 also inhibited hepatic stellate cell proliferation by targeting ZEB2 through Wnt/ $\beta$-catenin pathway (32). Combined with these results, we considered that the suppressive effect of miR-545 on the development of NSCLC was partly exhibited through the abnormal ZEB2 expression involved in Wnt/ $\beta$-catenin pathway.

In conclusion, downregulation of miR-545 was identified in NSCLC which is involved in tumorigenesis and prognosis of NSCLC. Moreover, ZEB2 was confirmed as a direct target gene of miR-545. miR-545 inhibited progression of NSCLC through targeting ZEB2 and blocking EMT and Wnt/ $\beta$-catenin pathway. These results can potentially help in elucidation of the regulatory mechanism of miR-545 in NSCLC.

\section{Acknowledgements}

Not applicable.

\section{Funding}

No funding was received.

\section{Availability of data and materials}

The datasets used during the present study are available from the corresponding author upon reasonable request.

\section{Authors' contributions}

JC contributed to the study design and drafted the manuscript. GP was involved in data acquisition and revision of the manuscript. QH was involved in data acquisition and analysis. LY contributed to data analysis. RG contributed to data acquisition and analysis and revised the manuscript. HB contributed to the conception and design of the study. All authors have read and approved the final manuscript.

\section{Ethics approval and consent to participate}

All the patients provided an informed consent and this experiment was approved by the Institutional Ethics Committee of The Third People's Hospital of Qingdao (Qingdao, China).

\section{Patient consent for publication}

Not applicable.

\section{Competing interests}

The authors declare that they have no competing interests.

\section{References}

1. Field JK, Oudkerk M, Pedersen JH and Duffy SW: Prospects for population screening and diagnosis of lung cancer. Lancet 382: 732-741, 2013.

2. Wang L, Yu C, Liu Y, Wang J, Li C, Wang Q, Wang P, Wu S and Zhang ZJ: Lung cancer mortality trends in China from 1988 to 2013: New challenges and opportunities for the government. Int J Environ Res Public Health 13: E1052, 2016.

3. Reck M, Popat S, Reinmuth N, De Ruysscher D, Kerr KM and Peters S; ESMO Guidelines Working Group: Metastatic non-small-cell lung cancer (NSCLC): ESMO Clinical Practice Guidelines for diagnosis, treatment and follow-up. Ann Oncol 25 (Suppl 3): iii27-iii39, 2014.

4. Pao W and Girard N: New driver mutations in non-small-cell lung cancer. Lancet Oncol 12: 175-180, 2011.

5. Araujo LH and Carbone DP: Non-small cell lung cancer genomics around the globe: Focus on ethnicity. J Thorac Dis 9: E392-E394, 2017.

6. Lv X, Li CY, Han P and Xu XY: MicroRNA-520a-3p inhibits cell growth and metastasis of non-small cell lung cancer through PI3K/AKT/mTOR signaling pathway. Eur Rev Med Pharmacol Sci 22: 2321-2327, 2018 
7. Fei X,Zhang J,Zhao Y, Sun M,Zhao H and Li S: miR-96 promotes invasion and metastasis by targeting GPC 3 in non-small cell lung cancer cells. Oncol Lett 15: 9081-9086, 2018.

8. Song B, Ji W, Guo S, Liu A, Jing W, Shao C, Li G and Jin G: miR-545 inhibited pancreatic ductal adenocarcinoma growth by targeting RIG-I. FEBS Lett 588: 4375-4381, 2014.

9. Cosín-Tomás M, Antonell A, Lladó A, Alcolea D, Fortea J, Ezquerra M, Lleó A, Martí MJ, Pallàs M, Sanchez-Valle R, et al: Plasma miR-34a-5p and miR-545-3p as early biomarkers of Alzheimer's disease: Potential and limitations. Mol Neurobiol 54: 5550-5562, 2017.

10. Du B, Wang Z, Zhang X, Feng S, Wang G, He J and Zhang B: MicroRNA-545 suppresses cell proliferation by targeting cyclin D1 and CDK4 in lung cancer cells. PLoS One 9: e88022, 2014.

11. Gheldof A, Hulpiau P, van Roy F, De Craene B and Berx G: Evolutionary functional analysis and molecular regulation of the ZEB transcription factors. Cell Mol Life Sci 69: 2527-2541, 2012

12. Chua HL, Bhat-Nakshatri P, Clare SE, Morimiya A, Badve S and Nakshatri H: NF-kappaB represses E-cadherin expression and enhances epithelial to mesenchymal transition of mammary epithelial cells: Potential involvement of ZEB-1 and ZEB-2. Oncogene 26: 711-724, 2007.

13. You J, Li Y, Fang N, Liu B, Zu L, Chang R, Li X and Zhou Q: MiR-132 suppresses the migration and invasion of lung cancer cells via targeting the EMT regulator ZEB2. PLoS One 9: e91827, 2014

14. Cong N, Du P, Zhang A, Shen F, Su J, Pu P, Wang T, Zjang J, Kang $C$ and Zhang Q: Downregulated microRNA-200a promotes EMT and tumor growth through the wnt/ $\beta$-catenin pathway by targeting the E-cadherin repressors ZEB1/ZEB2 in gastric adenocarcinoma. Oncol Rep 29: 1579-1587, 2013.

15. Zhang Q, Zhang B, Sun L, Yan Q, Zhang Y, Zhang Z, Su Y and Wang C: MicroRNA-130b targets PTEN to induce resistance to cisplatin in lung cancer cells by activating Wnt/ $\beta$-catenin pathway. Cell Biochem Funct 36: 194-202, 2018.

16. Livak KJ and Schmittgen TD: Analysis of relative gene expression data using real-time quantitative PCR and the 2(-Delta Delta C(T)) method. Methods 25: 402-408, 2001.

17. Brown BD and Naldini L: Exploiting and antagonizing microRNA regulation for therapeutic and experimental applications. Nat Rev Genet 10: 578-585, 2009.

18. Han L, Chen W, Xia Y, Song Y, Zhao Z, Cheng H and Jiang T: MiR-101 inhibits the proliferation and metastasis of lung cancer by targeting zinc finger E-box binding homeobox 1 . Am J Transl Res 10: 1172-1183, 2018.

19. Zhang W, Qiao B and Fan J: Overexpression of miR-4443 promotes the resistance of non-small cell lung cancer cells to epirubicin by targeting INPP4A and regulating the activation of JAK2/STAT3 pathway. Pharmazie 73: 386-392, 2018

20. Liao C, Xiao W, Zhu N, Liu Z, Yang J, Wang Y and Hong M: MicroR-545 enhanced radiosensitivity via suppressing Ku70 expression in Lewis lung carcinoma xenograft model. Cancer Cell Int 15: 56, 2015.
21. Liu Z, Dou C, Yao B, Xu M, Ding L, Wang Y, Jia Y, Li Q, Zhang H, Tu K, et al: Ftx non coding RNA-derived miR-545 promotes cell proliferation by targeting RIG-I in hepatocellular carcinoma. Oncotarget 7: 25350-25365, 2016.

22. Jia X, Liu X, Li M, Zeng Y, Feng Z, Su X, Huang Y, Chen M and Yang X: Potential tumor suppressing role of microRNA-545 in epithelial ovarian cancer. Oncol Lett 15: 6386-6392, 2018.

23. Huang $X$ and Lu S: MicroR-545 mediates colorectal cancer cells proliferation through up-regulating epidermal growth factor receptor expression in HOTAIR long non-coding RNA dependent. Mol Cell Biochem 431: 45-54, 2017.

24. Lin X, Yang Z, Zhang P, Liu Y and Shao G: miR-154 inhibits migration and invasion of human non-small cell lung cancer by targeting ZEB2. Oncol Lett 12: 301-306, 2016.

25. Fei D, Zhao K, Yuan H, Xing J and Zhao D: MicroRNA-187 exerts tumor-suppressing functions in osteosarcoma by targeting ZEB2. Am J Cancer Res 6: 2859-2868, 2016.

26. Jiao A, Sui M, Zhang L, Sun P, Geng D, Zhang W, Wang X and Li J: MicroRNA-200c inhibits the metastasis of non-small cell lung cancer cells by targeting ZEB2, an epithelial-mesenchymal transition regulator. Mol Med Rep 13: 3349-3355, 2016.

27. Comijn J, Berx G, Vermassen P, Verschueren K, van Grunsven L, Bruyneel E, Mareel M, Huylebroeck D and van Roy F: The two-handed $\mathrm{E}$ box binding zinc finger protein SIP1 downregulates E-cadherin and induces invasion. Mol Cell 7: 1267-1278, 2001.

28. Shi ZM, Wang L, Shen H, Jiang CF, Ge X, Li DM, Wen YY, Sun HR, Pan MH, Li W, et al: Downregulation of miR-218 contributes to epithelial-mesenchymal transition and tumor metastasis in lung cancer by targeting Slug/ZEB2 signaling. Oncogene 36: 2577-2588, 2017.

29. Qi S, Song Y, Peng Y, Wang H, Long H, Yu X, Li Z, Fang L, Wu A, Luo W, et al: ZEB2 mediates multiple pathways regulating cell proliferation, migration, invasion, and apoptosis in glioma. PLoS One 7: e38842, 2012.

30. Polakis P: Wnt signaling in cancer. Cold Spring Harb Perspect Biol 4: a008052, 2012

31. Wang XH, Cui YX, Wang ZM and Liu J: Down-regulation of FOXR2 inhibits non-small cell lung cancer cell proliferation and invasion through the Wnt $/ \beta$-catenin signaling pathway. Biochem Biophys Res Commun 500: 229-235, 2018.

32. Zhou DD, Wang X, Wang Y, Xiang XJ, Liang ZC, Zhou Y, Xu A, Bi CH and Zhang L: MicroRNA-145 inhibits hepatic stellate cell activation and proliferation by targeting ZEB2 through Wnt/ $\beta$-catenin pathway. Mol Immunol 75: 151-160, 2016.

This work is licensed under a Creative Commons Attribution-NonCommercial-NoDerivatives 4.0 International (CC BY-NC-ND 4.0) License. 